\title{
Editorial Message: Special Issue on Advances in Fuzzy Systems and Type-2 Fuzzy Sets
}

\author{
Jin-Tsong Jeng ${ }^{1} \cdot$ Byung-Jae Choi ${ }^{2}$
}

Published online: 24 January 2020

(C) Taiwan Fuzzy Systems Association 2020

In recent, advances in Fuzzy Systems and Type-2 Fuzzy Sets have been two of popular directions in artificial intelligence. Advances in Fuzzy Systems on this special issue have fuzzy mediation analysis, ridge fuzzy regression model, and a fuzzy approach to determining critical factors of diabetic retinopathy and enhancing data classification accuracy, FLS-based fuzzy synchronization control of complex dynamical networks, and fuzzy neural networks. Besides, Advances in Type-2 Fuzzy Sets have been successfully used in many areas, such as computing with words, human resource management, forecasting of time series, pattern recognition, fuzzy logic controller, industrial application, stock market index prediction, etc. Advances in Type-2 Fuzzy Sets on this special issue have long-term traffic volume prediction based on type-2 fuzzy sets with confidence interval method, an extended TOPSIS method based on Gaussian interval type- 2 fuzzy sets, and application of interval type-2 fuzzy sets in unmanned vehicle visual guidance. The objective of this special issue is to explore novel Advances in Fuzzy Systems and Type-2 Fuzzy Sets at the 2018 International Conference on Fuzzy Theory and Its Applications (iFUZZY 2018) held in EXCO, Daegu, Korea, over November 14-17, 2018. Papers submitted to the IJFS special issue were initially reviewed by the guest editor and accepted as the oral presentation. In accordance with the strict paper review procedure of IJFS, we invited IJFS associate editors, Prof. Shun-Feng Su (the current Editor-in-

Jin-Tsong Jeng

tsong@nfu.edu.tw

1 Department of Computer Science and Information Engineering, National Formosa University, Yunlin County, Taiwan

2 School of Electronic Engineering, Daegu University, Gyeongsan, Republic of Korea
Chief of IJFS), Prof. Ching-Chih Tsai, Prof. Jin-Tsong Jeng, Prof. Wen-June Wang, Prof. Youngchul Bae, and Prof. Byung-Jae Choi as the reviewers of all the oral presentations in iFUZZY 2018. These papers have oral presentation on a question-and-answer session with three reviewers and are open to all the audiences. Totally, seven papers were selected according to the review criterion on completeness and technical contributions and asked for quality improvements according to the reviewers' comments. Throughout the elaborative revisions by the authors, these seven papers have finally been accepted for publication in the IJFS special issue on fuzzy theory and its applications. As for the presented contents of these seven papers in the special issue, we make brief introductions to their contributions on Advances in Fuzzy Systems and Type-2 Fuzzy Sets that have one paper in this volume; these articles provide interesting and timely innovative results covering: Fuzzy Mediation Analysis. There are three papers in volume 21(6); these articles provide interesting and timely innovative results covering: Application of Interval Type-2 Fuzzy Sets in Unmanned Vehicle Visual Guidance, An Extended TOPSIS Method Based on Gaussian Interval Type-2 Fuzzy Sets, and A Fuzzy Approach to Determining Critical Factors of Diabetic Retinopathy and Enhancing Data Classification Accuracy. There are three papers in volume 21(7); these articles provide interesting and timely innovative results covering: Long-term Traffic Volume Prediction Based on Type-2 Fuzzy Sets with Confidence Interval Method, Ridge Fuzzy Regression Model, and Fuzzy Synchronization Control of Complex Dynamical Networks under Network Attacks and Actuator Faults. These seven papers bring a rich collection of Advances in Fuzzy Systems and Type-2 Fuzzy Sets to illustrate the main technical achievements on the IJFS special issue of iFUZZY 2018. Finally, I would like to acknowledge all the contributors to this special issue on the IJFS special issue of iFUZZY 2018. 